\title{
The eigenvalue problem for the 1-biharmonic operator
}

\author{
ENEA PARINI, BERNHARD RUF AND CRISTINA TARSI
}

\begin{abstract}
We consider the problem of finding the optimal constant for the embedding of the space

$$
W_{\Delta}^{2,1}(\Omega):=\left\{u \in W_{0}^{1,1}(\Omega) \mid \quad \Delta u \in L^{1}(\Omega)\right\}
$$

into the space $L^{1}(\Omega)$, where $\Omega \subseteq \mathbb{R}^{n}$ is a bounded convex domain, or a bounded domain with boundary of class $C^{1, \alpha}$. This is equivalent to finding the first eigenvalue of the 1-biharmonic operator under (generalized) Navier boundary conditions. In this paper we provide an interpretation for the eigenvalue problem, we show some properties of the first eigenfunction, we prove an inequality of FaberKrahn type, and we compute the first eigenvalue and the associated eigenfunction explicitly for a ball, and in terms of the torsion function for general domains.
\end{abstract}

Mathematics Subject Classification (2010): 46E35 (primary); 35G15, 35P30, 49J52 (secondary).

\section{Introduction}

Let $\Omega \subseteq \mathbb{R}^{n}$ be a bounded domain, and let $W_{\Delta}^{2,1}(\Omega)$ be defined as

$$
W_{\Delta}^{2,1}(\Omega):=\left\{u \in W_{0}^{1,1}(\Omega) \mid \quad \Delta u \in L^{1}(\Omega)\right\} .
$$

This function space turns out to be strictly larger than the Sobolev space $W^{2,1}(\Omega) \cap$ $W_{0}^{1,1}(\Omega)$, in which the whole set of second order derivatives is considered, in contrast with the case $p>1$ where we always have $W_{\Delta}^{2, p}(\Omega)=W^{2, p}(\Omega) \cap W_{0}^{1, p}(\Omega)$, provided $\partial \Omega$ is sufficiently smooth: the equivalence between the full Sobolev norm and $\|\Delta u\|_{p}$ can be achieved by standard elliptic theory, see [17, Lemma 9.17]. This difference is highlighted by the corresponding sharp Sobolev embeddings, in particular in the so-called limiting case $N=2\left(p=1=\frac{N}{2}\right)$ one has $W^{2,1}(\Omega) \hookrightarrow$ $L^{\infty}(\Omega)$ (see, e.g. [2]), while this embedding fails for the larger space $W_{\Delta}^{2,1}(\Omega)$, which embeds only into $L_{\exp }(\Omega)$ (see [7]).

Our aim here is to find the optimal constant for the embedding

$$
W_{\Delta}^{2,1}(\Omega) \hookrightarrow L^{1}(\Omega)
$$


and to study the properties of the extremal functions. The optimal constant is given by the inverse of the quantity

$$
\inf _{u \in W_{\Delta}^{2,1}(\Omega) \backslash\{0\}} \frac{\|\Delta u\|_{1}}{\|u\|_{1}} .
$$

Our approach is inspired by the minimization of the quantity

$$
\inf _{u \in W_{0}^{1,1}(\Omega) \backslash\{0\}} \frac{\|\nabla u\|_{1}}{\|u\|_{1}},
$$

for finding a first eigenfunction of the 1-Laplace operator (see [13,32]). Since the space $W_{0}^{1,1}(\Omega)$ is not reflexive, minimizing sequences of (1.2) may not converge weakly in $W_{0}^{1,1}(\Omega)$ : an appropriate space for the minimization problem is then $B V(\Omega)$, the space of the functions of bounded variation, and the existence of a minumum is a consequence of the lower semicontinuity of the total variation in $L_{\text {loc }}^{1}(\Omega)($ see $[3])$.

A minimizer of problem (1.2) formally solves the eigenvalue problem

$$
\begin{cases}-\operatorname{div}\left(\frac{\nabla u}{|\nabla u|}\right)=\lambda \frac{u}{|u|} & \text { in } \Omega \\ u=0 & \text { on } \partial \Omega .\end{cases}
$$

The nonlinear operator $\Delta u$ defined by

$$
\Delta_{1} u:=\operatorname{div}\left(\frac{\nabla u}{|\nabla u|}\right)
$$

is called the 1-Laplacian, and has been widely studied in recent years, due to its numerous applications (e.g. in control theory and game theory, in mathematical image restoration, in the theory of torsion and related geometric problems). Problem (1.3) can also be seen as the limiting case, for $p \rightarrow 1$, of the eigenvalue problem for the $p$-Laplace operator $\Delta_{p} u:=\operatorname{div}\left(|\nabla u|^{p-2} \nabla u\right)$ (see [19] and [27]); this interpretation justifies a purely theoretical interest in the study of (1.3), since, as pointed out by Evans ( [16]) in an article on the 1-Laplacian and the $\infty$-Laplacian: "an important principle of mathematics is that extreme cases reveal interesting structures".

Motivated by these considerations, we aim to study the 'higher-order' case, namely the minimization problem (1.1). As for the first eigenvalue of the 1-Laplacian, the infimum is not attained in $W_{\Delta}^{2,1}(\Omega)$, and it will be necessary to consider the larger space $B L_{0}(\Omega)$, consisting of all functions $u \in W_{0}^{1,1}(\Omega)$ such that $\Delta u$ is a Radon measure with finite total variation: the first part of the paper (Sections 2-5) is devoted to the introduction of this new function space and its properties. Note that $B L_{0}(\Omega)$ contains properly $B H(\Omega)$, the space of functions with bounded Hessian, introduced in [12], and its subspace $S B H(\Omega)$ of functions 
with special bounded Hessian, which finds application in the elastic-plastic plate theory.

In order to make the minimization problem (1.1) well-posed, we require a regularity assumption on the domain $\Omega$ : if $\Omega$ is convex, or has a boundary of class $C^{1, \alpha}$, passing through the notion of solution in the sense of Stampacchia, we are able to guarantee that any function $u \in W_{0}^{1,1}(\Omega)$ harmonic in distributional sense is identically 0 . This property does not hold for general domains, as shown by a counterexample (see section 5).

Analogously to the case of problem (1.2), a minimizer of (1.1) formally satisfies

$$
\begin{cases}\Delta\left(\frac{\Delta u}{|\Delta u|}\right)=\lambda \frac{u}{|u|} & \text { in } \Omega \\ u=0 & \text { on } \partial \Omega \\ \frac{\Delta u}{|\Delta u|}=0 & \text { on } \partial \Omega .\end{cases}
$$

The operator

$$
\Delta_{1}^{2} u:=\Delta\left(\frac{\Delta u}{|\Delta u|}\right)
$$

can be seen as the limiting case, for $p \rightarrow 1$, of the $p$-biharmonic operator

$$
\Delta_{p}^{2} u:=\Delta\left(|\Delta u|^{p-2} \Delta u\right),
$$

and so we refer to it as the 1-biharmonic operator. The eigenvalue problem for the $p$-biharmonic operator under Navier boundary conditions (that is, $u=\Delta u=0$ ) was studied in [14], where it was proved that there exists a unique eigenfunction (up to nonzero multiplicative constants), which moreover can be chosen positive and superharmonic.

The rigorous derivation of the Euler-Lagrange problem (1.4) requires methods of nonsmooth analysis, due to the nonsmoothness of the involved functionals; furthermore, the quantities $\frac{\Delta u}{|\Delta u|}$ and $\frac{u}{|u|}$ in (1.4) are not well defined, so they must be interpreted in a generalized sense. Following the approach used in $[20,32]$ for the 1-Laplacian, for any measurable selection $s(x)$ of the set-valued sign function $\operatorname{Sgn}(u)$ we find some $z \in W_{0}^{1,1}(\Omega) \cap L^{\infty}(\Omega)$ such that

$$
\Delta z=\lambda s \quad \text { a.e. on } \Omega, \quad \text { with } \lambda=\frac{|\Delta u|_{T}}{\|u\|_{1}} .
$$

The function $z$ can be considered as a substitute of $\Delta u /|\Delta u|$ in the formal 1biharmonic operator, while $s$ substitutes the formal multiplier $u /|u|$ at points where $u$ vanishes. Hence, it turns out that for minimizers $u \in B L_{0}(\Omega)$ of (1.1) infinitely many Euler Lagrange equations have to be satisfied, in general.

Using standard symmetrization techniques, a Faber-Krahn inequality for the 1-Laplacian can be easily derived, which states that, among all domains with prescribed volume, the first eigenvalue is minimal for the ball: the proof relies on the 
Pólya-Szegö inequality, which assures that the $L^{p}$ norm of the gradient of a function $u$ in $W_{0}^{1, p}(\Omega)(1 \leq p \leq \infty)$ does not increase under symmetrization. On the contrary, dealing with second order derivatives, a Pólya-Szegö type result is not available, and the derivation of a Faber-Krahn inequality for the 1-biharmonic operator is no longer a trivial task. Nevertheless, we are able to prove it using in particular a comparison result due to Talenti.

We are now ready to state our main theorem which summarizes the results described above.

Theorem 1.1. Let $\Omega \subseteq \mathbb{R}^{n}$ be a bounded convex domain, or a bounded domain with boundary of class $C^{1, \alpha}(0<\alpha \leq 1)$, and let

$$
B L_{0}(\Omega):=\left\{\left.u \in W_{0}^{1,1}(\Omega)|\quad| \Delta u\right|_{T}<\infty\right\}
$$

where $|\Delta u|_{T}$ denotes the total variation of the distributional Laplacian $\Delta u$. Consider the minimization problem

$$
\Lambda_{1,1}(\Omega):=\inf _{u \in B L_{0}(\Omega) \backslash\{0\}} \frac{|\Delta u|_{T}}{\|u\|_{1}} .
$$

Then:

(i) The infimum is attained: there is $v \in B L_{0}(\Omega):|\Delta v|_{T}=\Lambda_{1,1}(\Omega)\|v\|_{1}$; furthermore, $v$ is non-negative and superharmonic;

(ii) The minimizer formally satisfies

$$
\begin{cases}\Delta_{1}^{2} u=\lambda \frac{u}{|u|} & \text { in } \Omega \\ u=\frac{\Delta u}{|\Delta u|}=0 & \text { on } \partial \Omega\end{cases}
$$

in the sense that for any measurable sign selection $s \in S g n(u)$ there exists $z \in W_{0}^{1,1}(\Omega) \cap L^{\infty}(\Omega)$ such that

- $\|z\|_{\infty}=1, \Delta z \in L^{n}(\Omega)$;

- $|\Delta u|_{T}=\int_{\Omega} u \Delta z$;

- $\Delta z=\lambda$ s almost everywhere in $\Omega$, with $\lambda=\frac{|\Delta u|_{T}}{\|u\|_{1}}$.

The following result provides a physical interpretation of $\Lambda_{1,1}(\Omega)$ : the first eigenvalue can be computed in terms of the torsion function and, in the case of radial domains, its value can be explicitly exhibited, as well as the corresponding first eigenfunction.

Theorem 1.2. For any convex domain $\Omega \subseteq \mathbb{R}^{n}$, or any domain with $C^{1, \alpha}$ boundary $(0<\alpha \leq 1)$, the first eigenvalue $\Lambda_{1,1}(\Omega)$ of the 1-biharmonic operator is given by

$$
\Lambda_{1,1}(\Omega)=\frac{1}{\psi_{M}(\Omega)}, \quad \psi_{M}(\Omega)=\max _{x \in \Omega} \psi(x)
$$


where $\psi$ is the solution of the torsion problem

$$
\begin{cases}-\Delta \psi=1 & \text { in } \Omega \\ \psi=0 & \text { on } \partial \Omega .\end{cases}
$$

Furthermore, the Green function $G\left(z_{M}, x\right)$, for the domain $\Omega$, provides a corresponding eigenfunction, for any $z_{M} \in \Omega$, maximum point of $\psi$ (i.e. $\psi_{M}(\Omega)=$ $\left.\psi\left(z_{M}\right)\right)$.

If $\Omega=B_{R}$ the ball of radius $R$ centered in the origin, then

$$
\Lambda_{1,1}\left(B_{R}\right)=\frac{2 n}{R^{2}}
$$

and a first eigenfunction is given by the solution of

$$
\begin{cases}-\Delta u=\delta_{0} & \text { in } B_{R} \\ u=0 & \text { on } \partial B_{R},\end{cases}
$$

where $\delta_{0}$ is a Dirac mass concentrated in 0 .

Finally, the following Faber-Krahn type result holds true for the 1-biharmonic operator:

Theorem 1.3. Let $\Omega \subseteq \mathbb{R}^{n}$ be a bounded convex domain, or a bounded domain with boundary of class $C^{1, \alpha}(0<\alpha \leq 1)$; then

$$
\Lambda_{1,1}\left(\Omega^{\#}\right) \leq \Lambda_{1,1}(\Omega)
$$

where $\Omega^{\#}$ is a ball with $\left|\Omega^{\#}\right|=|\Omega|$. Moreover, equality holds if and only if $\Omega$ is a ball.

Since under dilation the first eigenvalue $\Lambda_{1,1}(\Omega)$ scales as

$$
\Lambda_{1,1}(t \Omega)=\frac{1}{t^{2}} \Lambda_{1,1}(\Omega),
$$

the Faber-Krahn type inequality can be written in the scaling-invariant form

$$
|\Omega|^{2 / n} \Lambda_{1,1}(\Omega) \geq 2 n \omega_{n}^{2 / n}
$$

where $\omega_{n}=\pi^{n / 2} / \Gamma(1+n / 2)$ is the Lebesgue measure of the $n$-dimensional unit ball (see Remark 6.7).

The paper is organized as follows: after giving the necessary definitions and proving some preliminary results in Section 2, we give an approximation result for functions in $B L_{0}(\Omega)$ (Section 3). In the fourth section we discuss the wellposedness of our minimization problem, while in Section 5 we prove Theorem 1.1. 
Finally, Section 6 provides a physical interpretation of the first eigenvalue (Theorem 1.2) as well as a Faber-Krahn type inequality (Theorem 1.3).

ACKNOWLEDGements. The authors would like to thank Guido Sweers and Athanasios Stylianou for interesting and useful discussions about higher-order problems, and in particular about the counterexample in Section 5. We are also grateful to Guido De Philippis for his observations that led to the proof of Proposition 6.6, and to Lorenzo Brasco for suggestions about how to improve the regularity assumptions in Proposition 4.3. Finally, we wish to thank the Referee for the careful reading of the manuscript and the useful remarks that have helped us revise and improve the article.

\section{Definitions and preliminary results}

Let $\Omega \subseteq \mathbb{R}^{n}$ be a bounded domain; unless otherwise specified, we will suppose that its boundary is of Lipschitz class. A function $u \in L^{1}(\Omega)$ is said to be of bounded variation if its distributional derivative is representable by a finite Radon measure $\mu$, i.e. if

$$
\int_{\Omega} u \operatorname{div} \varphi=\int_{\Omega} \varphi d \mu \quad \forall \varphi \in C_{c}^{\infty}\left(\Omega ; \mathbb{R}^{n}\right),
$$

or, equivalently, if the quantity

$$
|D u|:=\sup \left\{\int_{\Omega} u \operatorname{div} \varphi \mid \varphi \in C_{c}^{\infty}\left(\Omega ; \mathbb{R}^{n}\right),\|\varphi\|_{\infty} \leq 1\right\}
$$

is finite. The space of functions of bounded variation is denoted by $B V(\Omega)$. Analogously, we define $B L(\Omega)$ as the space of functions $u \in W^{1,1}(\Omega)$ whose Laplacian $\Delta u$ is representable by a finite measure $\mu$, i.e.

$$
\int_{\Omega} \nabla u \nabla \varphi=\int_{\Omega} \varphi d \mu \quad \forall \varphi \in C_{c}^{\infty}(\Omega ; \mathbb{R}) .
$$

Recalling that the total variation of a measure is defined as

$$
|\mu|(\Omega)=\sup \left\{\int_{\Omega} \varphi d \mu \mid \varphi \in C_{c}(\Omega),\|\varphi\|_{\infty} \leq 1\right\}
$$

we define the total variation of the Laplacian of $u$ to be the quantity

$$
\begin{aligned}
|\Delta u|_{T} & :=\sup \left\{\int_{\Omega} \varphi d \Delta u \mid \varphi \in C_{c}(\Omega),\|\varphi\|_{\infty} \leq 1\right\} \\
& =\sup \left\{\int_{\Omega} \varphi d \Delta u \mid \varphi \in C_{c}^{\infty}(\Omega),\|\varphi\|_{\infty} \leq 1\right\}
\end{aligned}
$$


where we used the fact that $C_{c}^{\infty}(\Omega)$ is dense in $C_{c}(\Omega)$. Hence

$$
B L(\Omega)=\left\{\left.u \in W^{1,1}(\Omega)|\quad| \Delta u\right|_{T}<\infty\right\} .
$$

Furthermore, we denote by $B L_{0}(\Omega)$ the space

$$
B L_{0}(\Omega):=\left\{\left.u \in W_{0}^{1,1}(\Omega)|\quad| \Delta u\right|_{T}<\infty\right\} .
$$

For $n=1$, the space $B L(\Omega)$ coincides with the space of functions of bounded Hessian $B H(\Omega)$ introduced in [12], which are functions whose gradient is locally in $B V(\Omega)$. However, if $n \geq 2$ the latter space is strictly contained in $B L(\Omega)$, as a consequence of the results in [10, Theorem 3]; indeed, the authors prove the existence of a function $u:[0,1] \times[0,1] \rightarrow \mathbb{R}$ such that $u_{x x}$ and $u_{y y}$ are Radon measures with finite total variation, but the total variation of $u_{x y}$ is infinite, so that $u \notin B H(\Omega)$. Finally, we define the space

$$
W_{\Delta}^{2,1}(\Omega):=\left\{u \in W_{0}^{1,1}(\Omega) \mid \quad \Delta u \in L^{1}(\Omega)\right\} .
$$

Note that $W_{\Delta}^{2,1}(\Omega) \subseteq B L_{0}(\Omega)$ : indeed, for any $u \in W_{\Delta}^{2,1}(\Omega)$ the distributional Laplacian is given by $\Delta u d x$, but the inclusion is strict: there exist functions $u \in$ $B L(\Omega)$ such that $\Delta u$ is singular with respect to the Lebesgue measure. As an example, one can consider the Green function of $\Omega$ defined as

$$
\begin{cases}-\Delta u=\delta_{y} & \text { in } \Omega \\ u=0 & \text { on } \partial \Omega\end{cases}
$$

where $\delta_{y}$ is a Dirac mass concentrated in a point $y \in \Omega$. Then $u \in B L_{0}(\Omega)$, but $u \notin W_{\Delta}^{2,1}(\Omega)$. Furthermore, if $u \in W_{\Delta}^{2,1}(\Omega)$, then $|\Delta u|_{T}=\|\Delta u\|_{1}$. Indeed one has

$$
|\Delta u|_{T}:=\sup \left\{\int_{\Omega} \varphi \Delta u \mid \varphi \in C_{c}(\Omega), \quad\|\varphi\|_{\infty} \leq 1\right\}
$$

so that, for any admissible function $\varphi$,

$$
\left|\int_{\Omega} \Delta u \varphi\right| \leq \int_{\Omega}|\Delta u||\varphi| \leq \int_{\Omega}|\Delta u|=\|\Delta u\|_{1},
$$

which implies $|\Delta u|_{T} \leq\|\Delta u\|_{1}$.

To prove the reverse inequality, let us denote by $\psi$ the function $\psi:=\operatorname{sgn}(\Delta u)$ and by $\psi_{k}$ the function $\psi_{k}:=\psi \chi_{\Omega_{k}}$ where

$$
\Omega_{k}:=\left\{x \in \Omega \mid \operatorname{dist}(x, \partial \Omega)>\frac{1}{k}\right\} .
$$


Since $\psi_{k}$ is measurable and has compact support, by Lusin's Theorem there exists a function $\varphi_{k} \in C_{c}(\Omega)$ such that $\left\|\varphi_{k}\right\|_{\infty} \leq\left\|\psi_{k}\right\|_{\infty}=1$ and

$$
V_{k}:=\left|\left\{\varphi_{k} \neq \psi_{k}\right\}\right| \leq \frac{1}{k} \text {. }
$$

Hence

$$
\begin{aligned}
\left|\int_{\Omega} \Delta u \varphi_{k}-\|\Delta u\|_{1}\right| & =\left|\int_{\Omega_{k}} \Delta u\left(\varphi_{k}-\psi\right)+\int_{\Omega \backslash \Omega_{k}} \Delta u\left(\varphi_{k}-\psi\right)\right| \\
& \leq\left|\int_{\Omega_{k}} \Delta u\left(\varphi_{k}-\psi_{k}\right)\right|+\left|\int_{\Omega \backslash \Omega_{k}} \Delta u\left(\varphi_{k}-\psi\right)\right| \\
& \leq 2 \int_{V_{k} \cap \Omega_{k}}|\Delta u|+2 \int_{\Omega \backslash \Omega_{k}}|\Delta u| \rightarrow 0
\end{aligned}
$$

as $k \rightarrow \infty$. From the definition of $|\Delta u|_{T}$ it follows that $\|\Delta u\|_{1} \leq|\Delta u|_{T}$. This yields the claim.

Remark 2.1. The quantity $|\Delta u|_{T}$ is lower semicontinuous with respect to the $L^{1}$ convergence. To check this, we need only to notice that $|\Delta u|_{T}$ is the supremum of the functionals $T_{\varphi}$ defined for $\varphi \in C_{c}^{\infty}(\Omega)$ as

$$
T_{\varphi}(u)=\int_{\Omega} u \Delta \varphi d x,
$$

which are continuous in the $L^{1}(\Omega)$ topology.

Finally, we present an equivalent definition of the space $B L(\Omega)$, which will be used in the proof of Proposition 3.1.

Proposition 2.2. Let $u \in W^{1,1}(\Omega)$. Then $u \in B L(\Omega)$ if and only if

$$
\begin{aligned}
V_{\Delta}(u, \Omega) & =\sup \left\{\int_{\Omega} \nabla u \nabla \varphi \mid \varphi \in C_{c}^{\infty}(\Omega ; \mathbb{R}),\|\varphi\|_{\infty} \leq 1\right\} \\
& =\sup \left\{\int_{\Omega} u \Delta \varphi \mid \varphi \in C_{c}^{\infty}(\Omega ; \mathbb{R}),\|\varphi\|_{\infty} \leq 1\right\}<+\infty .
\end{aligned}
$$

Moreover, $V_{\Delta}(u, \Omega)=|\Delta u|_{T}$.

Proof. Suppose that there exists a Radon measure $\mu$ with finite total variation such that $-\Delta u=\mu$ in distributional sense. Then

$$
\begin{aligned}
V_{\Delta}(u, \Omega) & =\sup \left\{\int_{\Omega} \nabla u \nabla \varphi \mid \quad \varphi \in C_{c}^{\infty}(\Omega),\|\varphi\|_{\infty} \leq 1\right\} \\
& =\sup \left\{\int_{\Omega} \varphi d \mu \mid \varphi \in C_{c}^{\infty}(\Omega),\|\varphi\|_{\infty} \leq 1\right\} \\
& =\sup \left\{\int_{\Omega} \varphi d \mu \mid \varphi \in C_{c}(\Omega),\|\varphi\|_{\infty} \leq 1\right\}=|\Delta u|_{T}(\Omega),
\end{aligned}
$$


where we used the fact that $C_{c}^{\infty}(\Omega)$ is dense in $C_{c}(\Omega)$. Conversely, suppose that $V_{\Delta}(u, \Omega)<+\infty$. Then

$$
\int_{\Omega} \nabla u \nabla \varphi \leq V_{\Delta}(u, \Omega) \cdot\|\varphi\|_{\infty}
$$

for every $\varphi \in C_{c}^{\infty}(\Omega)$. Since $C_{c}^{\infty}(\Omega)$ is dense in $C_{c}(\Omega)$, we can find a linear continuous operator $L$ on $C_{0}(\Omega)$ such that $L \varphi=\int_{\Omega} \nabla u \nabla \varphi$ for every $\varphi \in C_{c}^{\infty}(\Omega)$, and $\|L\| \leq V_{\Delta}(u, \Omega)$.

By the Riesz representation Theorem (see [31, Theorem 6.19]) there exists a Radon measure $\mu$ with $\|L\|=|\mu|(\Omega)$ and $L \varphi=\int_{\Omega} \varphi d \mu$ for every $\varphi \in C_{c}(\Omega)$. In particular $\int_{\Omega} \nabla u \nabla \varphi=\int_{\Omega} \varphi d \mu$ for every $\varphi \in C_{c}^{\infty}(\Omega)$, which means $-\Delta u=\mu$ in distributional sense and implies

$$
V_{\Delta}(u, \Omega)=|\Delta u|_{T} .
$$

\section{An approximation result}

We say that a sequence $\left\{u_{k}\right\}$ in $B L(\Omega)$ converges strictly to $u \in B L(\Omega)$ if

- $u_{k} \rightarrow u$ in $W^{1,1}(\Omega)$ as $k \rightarrow \infty$, and

- $\left|\Delta u_{k}\right|_{T} \rightarrow|\Delta u|_{T}$ as $k \rightarrow \infty$.

In this section we prove that any function $B L(\Omega)$ can be approximated with respect to the strict convergence by a sequence of smooth functions. The proof is similar to the analogous result for $B V$ functions proved in [18, Theorem 1.17].

Proposition 3.1. Let $u \in B L(\Omega)$. Then there exists a sequence of functions $u_{k} \in$ $C^{\infty}(\Omega) \cap B L(\Omega)$ converging strictly to $u$ as $k \rightarrow \infty$.

Proof. Fix $\varepsilon>0$. There exists a number $m \in \mathbb{N}$ such that, if one sets for $k=$ $0,1,2 \ldots$

$$
\Omega_{k}:=\left\{x \in \Omega \mid \operatorname{dist}(x, \partial \Omega)>\frac{1}{m+k}\right\},
$$

then $|\Delta u|_{T}\left(\Omega \backslash \Omega_{0}\right)<\varepsilon$.

Define for $i \in \mathbb{N}$ the sets $A_{i}$ by $A_{1}:=\Omega_{2}$, and $A_{i}:=\Omega_{i+1} \backslash \bar{\Omega}_{i-1}$ for $i \geq 2$. Let $\left\{\psi_{i}\right\}_{i=1}^{\infty}$ be a partition of the unity subordinate to the covering $\left\{A_{i}\right\}$, which means that $\psi_{i} \in C_{0}^{\infty}\left(A_{i}\right), 0 \leq \psi_{i} \leq 1$, and $\sum_{i=1}^{\infty} \psi_{i} \equiv 1$.

Let $\eta$ be a positive symmetric mollifier. For every $i$, it is possible to choose $\varepsilon_{i}>0$ such that, setting $\eta_{i}:=\eta_{\varepsilon_{i}}$, one has:

- $\operatorname{supp} \eta_{i} *\left(u \psi_{i}\right) \subseteq \Omega_{i+2} \backslash \Omega_{i-2}\left(\right.$ where $\left.\Omega_{-1}:=\emptyset\right)$

- $\int_{\Omega}\left|\eta_{i} *\left(u \psi_{i}\right)-u \psi_{i}\right|<2^{-i} \varepsilon$

- $\int_{\Omega}\left|\eta_{i} * \nabla\left(u \psi_{i}\right)-\nabla\left(u \psi_{i}\right)\right|<2^{-i} \varepsilon$ 
- $\int_{\Omega}\left|\eta_{i} * \nabla u \nabla \psi_{i}-\nabla u \nabla \psi_{i}\right|<2^{-i} \varepsilon$

- $\int_{\Omega}\left|\eta_{i} * \operatorname{div}\left(u \nabla \psi_{i}\right)-\operatorname{div}\left(u \nabla \psi_{i}\right)\right|<2^{-i} \varepsilon$.

Define now $u_{\varepsilon}:=\sum_{i=1}^{\infty} \eta_{i} *\left(u \psi_{i}\right)$.

Since this sum is locally finite, it follows that $u_{\varepsilon} \in C^{\infty}(\Omega)$; furthermore, one has

$$
\int_{\Omega}\left|u_{\varepsilon}-u\right| \leq \sum_{i=1}^{\infty} \int_{\Omega}\left|\eta_{i} *\left(u \psi_{i}\right)-u \psi_{i}\right|<\varepsilon,
$$

and

$$
\int_{\Omega}\left|\nabla u_{\varepsilon}-\nabla u\right| \leq \sum_{i=1}^{\infty} \int_{\Omega}\left|\eta_{i} * \nabla\left(u \psi_{i}\right)-\nabla\left(u \psi_{i}\right)\right|<\varepsilon .
$$

Hence $u_{\varepsilon} \rightarrow u$ in $W_{0}^{1,1}(\Omega)$ as $\varepsilon \rightarrow 0$. It follows that $|\Delta u|_{T} \leq \liminf _{\varepsilon \rightarrow 0}\left|\Delta u_{\varepsilon}\right|_{T}$.

Let now $\varphi \in C_{c}^{\infty}(\Omega),\|\varphi\|_{\infty} \leq 1$. It holds

$$
\begin{aligned}
\int_{\Omega} \nabla u_{\varepsilon} \nabla \varphi= & \sum_{i=1}^{\infty} \int_{\Omega} \nabla\left(\eta_{i} * u \psi_{i}\right) \nabla \varphi=\sum_{i=1}^{\infty} \int_{\Omega}\left(\eta_{i} * \nabla\left(u \psi_{i}\right)\right) \nabla \varphi \\
= & \sum_{i=1}^{\infty} \int_{\Omega} \nabla\left(u \psi_{i}\right) \nabla\left(\eta_{i} * \varphi\right)=\sum_{i=1}^{\infty} \int_{\Omega} \psi_{i} \nabla u \nabla\left(\eta_{i} * \varphi\right) \\
& +\sum_{i=1}^{\infty} \int_{\Omega} u \nabla \psi_{i} \nabla\left(\eta_{i} * \varphi\right) \\
= & \sum_{i=1}^{\infty} \int_{\Omega} \nabla u \nabla\left(\psi_{i}\left(\eta_{i} * \varphi\right)\right)-\sum_{i=1}^{\infty} \int_{\Omega} \varphi\left(\eta_{i} * \nabla u \nabla \psi_{i}\right) \\
& -\sum_{i=1}^{\infty} \int_{\Omega} \operatorname{div}\left(u \nabla \psi_{i}\right)\left(\eta_{i} * \varphi\right) \\
= & \sum_{i=1}^{\infty} \int_{\Omega} \nabla u \nabla\left(\psi_{i}\left(\eta_{i} * \varphi\right)\right)-\sum_{i=1}^{\infty} \int_{\Omega} \varphi\left(\eta_{i} * \nabla u \nabla \psi_{i}\right) \\
& -\sum_{i=1}^{\infty} \int_{\Omega} \varphi\left(\eta_{i} * \operatorname{div}\left(u \nabla \psi_{i}\right)\right) \\
= & I_{1}+I_{2}+I_{3} .
\end{aligned}
$$

Let us analyze the three integrals separately. Since $\left|\psi_{i}\left(\eta_{i} * \varphi\right)\right| \leq 1$, one has, taking into account the fact that each point in $\Omega$ belongs at most to three of the sets $A_{i}$,

$$
\begin{aligned}
I_{1}= & \int_{\Omega} \nabla u \nabla\left(\psi_{1}\left(\eta_{1} * \varphi\right)\right) \\
& +\sum_{i=2}^{\infty} \int_{\Omega} \nabla u \nabla\left(\psi_{i}\left(\eta_{i} * \varphi\right)\right) \leq|\Delta u|_{T}+3|\Delta u|_{T}\left(\Omega \backslash \Omega_{0}\right) \leq|\Delta u|_{T}+3 \varepsilon .
\end{aligned}
$$


Recalling that $\sum_{i=1}^{\infty} \psi_{i} \equiv 1$ we obtain

$$
\begin{aligned}
\left|I_{2}\right| & =\left|\sum_{i=1}^{\infty} \int_{\Omega} \varphi\left(\eta_{i} * \nabla u \nabla \psi_{i}\right)\right|=\left|\sum_{i=1}^{\infty} \int_{\Omega} \varphi\left(\eta_{i} * \nabla u \nabla \psi_{i}-\nabla u \nabla \psi_{i}\right)\right| \\
& \leq \sum_{i=1}^{\infty} \int_{\Omega}\left|\eta_{i} * \nabla u \nabla \psi_{i}-\nabla u \nabla \psi_{i}\right|<\varepsilon .
\end{aligned}
$$

Finally

$$
\begin{aligned}
\left|I_{3}\right| & =\left|\sum_{i=1}^{\infty} \int_{\Omega} \varphi \eta_{i} * \operatorname{div}\left(u \nabla \psi_{i}\right)\right|=\left|\sum_{i=1}^{\infty} \int_{\Omega} \varphi\left(\eta_{i} * \operatorname{div}\left(u \nabla \psi_{i}\right)-\operatorname{div}\left(u \nabla \psi_{i}\right)\right)\right| \\
& \leq \sum_{i=1}^{\infty} \int_{\Omega}\left|\eta_{i} * \operatorname{div}\left(u \nabla \psi_{i}\right)-\operatorname{div}\left(u \nabla \psi_{i}\right)\right|<\varepsilon .
\end{aligned}
$$

Summing up we obtain $\left|\Delta u_{\varepsilon}\right|_{T} \leq|\Delta u|_{T}+5 \varepsilon$. Hence we obtain the claim.

As in [18, Remark 1.18], the approximating sequence $u_{k}$ can be chosen such that the trace of $u_{k}$ on $\partial \Omega$ coincides with the trace of $u$. Therefore we can state the following result.

Proposition 3.2. Let $u \in B L_{0}(\Omega)$. Then there exists a sequence of functions $u_{k} \in$ $C^{\infty}(\Omega) \cap C(\bar{\Omega}) \cap B L_{0}(\Omega)$ converging strictly to $u$ as $k \rightarrow \infty$.

Notice that $u \in B L_{0}(\Omega) \cap C^{\infty}(\Omega) \cap C(\bar{\Omega})$ implies $u \in W_{\Delta}^{2,1}(\Omega)$. Hence, as a consequence of Propositions 2.2 and 3.2 we obtain

$$
\inf _{u \in W_{\Delta}^{2,1}(\Omega) \backslash\{0\}} \frac{\|\Delta u\|_{1}}{\|u\|_{1}}=\inf _{u \in B L_{0}(\Omega) \backslash\{0\}} \frac{|\Delta u|_{T}}{\|u\|_{1}} .
$$

\section{Equivalence of distributional solutions and solutions in the sense of Stampacchia}

As remarked at the end of the previous section, we are led to study the minimization problem

$$
\inf _{u \in B L_{0}(\Omega) \backslash\{0\}} \frac{|\Delta u|_{T}}{\|u\|_{1}}
$$

in the broader space $B L_{0}(\Omega)$, that is, in the space of $W_{0}^{1,1}$-functions whose distributional Laplacian is a Radon measure. This new setting naturally leads to examine the properties of the equation

$$
\begin{cases}-\Delta u=\mu & \text { in } \Omega \\ u=0 & \text { on } \partial \Omega\end{cases}
$$


where $\mu$ is a Radon measure with finite bounded variation: in particular, we are interested in a suitable definition of solution for which we can guarantee existence and uniqueness. Problems involving elliptic equations with measure data have been widely studied by many authors: the linear case has been approached by Stampacchia in [35] by means of duality methods, whereas the general case has been considered, for example, in $[4,5,11]$ and, more recently, in [26].

The notion of distributional solution turns out to be too weak to approach the problem, as pointed out by Serrin: in [33] he exhibited a nontrivial family of functions $u_{\varepsilon}$, depending on a small parameter $\varepsilon$, which are distributional solutions of the problems

$$
\left\{\begin{aligned}
-\operatorname{div}\left(A_{\varepsilon}(x) \nabla u\right) & =0 & & x \in B \\
u & =0 & & x \in \partial B
\end{aligned}\right.
$$

where $A_{\varepsilon}(x)$ is a suitable family of bounded and coercive matrices. The distributional solutions $u_{\varepsilon}$ belong to $W_{0}^{1, \frac{n}{n-1}-\varepsilon}(B)$, but not to $H_{0}^{1}(B)$. On the other hand, the equations clearly have also weak solutions $v_{\varepsilon} \in H_{0}^{1}(B)$.

In the case of the Poisson equation, we can consider a simpler counterexample: let $\Omega \subseteq \mathbb{R}^{2}$ be defined in polar coordinates as

$$
\Omega=\left\{(r, \varphi) \in \mathbb{R}^{2} \mid 0<r<1,0<\varphi<\frac{3}{2} \pi\right\}
$$

and consider the function

$$
u(r, \varphi)=\left(r^{\frac{2}{3}}-r^{-\frac{2}{3}}\right) \cdot \sin \left(\frac{2}{3} \varphi\right) .
$$

It is easy to verify that $u \in W_{0}^{1,1}(\Omega), u \in C^{\infty}(\Omega)$ and $\Delta u=0$ in the classical (and hence distributional) sense; however, $u \neq \equiv 0$. This implies in particular that for this domain

$$
\inf _{v \in B L_{0}(\Omega) \backslash\{0\}} \frac{|\Delta v|_{T}}{\|v\|_{1}}=0 .
$$

Therefore we need to find some conditions on the domain $\Omega$ in order to guarantee uniqueness of the distributional solution of (4.1).

To this aim, we introduce a notion of weak solution due to Stampacchia [35] (Definition 9.1), that guarantees existence and uniqueness properties for (4.1); then, we will prove that solutions in the sense of Stampacchia and distributional solutions actually coincide, under suitable regularity assumptions on the boundary of $\Omega$.

Let $\mu$ be a Radon measure with finite bounded variation.

Definition 4.1. We say that $u$ is a solution in the sense of Stampacchia of

$$
\begin{cases}-\Delta u=\mu & \text { in } \Omega \\ u=0 & \text { on } \partial \Omega\end{cases}
$$


if

$$
-\int_{\Omega} u \Delta \varphi=\int_{\Omega} \varphi d \mu
$$

for every $\varphi \in W_{0}^{1,2}(\Omega) \cap C(\bar{\Omega})$ such that $\Delta \varphi \in C(\bar{\Omega})$.

It is clear that every solution in the sense of Stampacchia is a distributional solution of the same equation. By [35, Theorem 9.1], there exists exactly one solution $u$ in the sense of Stampacchia of (4.1), which moreover satisfies $u \in W_{0}^{1, r}(\Omega)$ for every $r \in\left[1, \frac{n}{n-1}\right)$. We have indeed the following:

Theorem 4.2 (Stampacchia). Let $\Omega$ be a domain with a Lipschitz boundary, and let $\mu$ be a Radon measure with finite total variation. Then there exists exactly one solution (in the sense of Stampacchia) of the problem

$$
\begin{cases}-\Delta u=\mu & \text { in } \Omega \\ u=0 & \text { on } \partial \Omega .\end{cases}
$$

The solution satisfies $u \in W_{0}^{1, r}(\Omega)$ for $r \in\left[1, \frac{n}{n-1}\right)$, and there is a constant $c(r)>$ 0 such that

$$
\|u\|_{W_{0}^{1, r}} \leq c(r)|\mu|_{T}
$$

where $|\mu|_{T}$ denotes the total variation of $\mu$.

Let us now prove that the notions of distributional solution and solution in the sense of Stampacchia coincide, provided $\Omega$ is a convex domain, or a domain with a boundary of class $C^{1, \alpha}$ with $\alpha \in(0,1]$.

Proposition 4.3. Let $\Omega$ be a bounded, convex domain or a bounded domain whose boundary is of class $C^{1, \alpha}$ with $\alpha \in(0,1]$. Then every distributional solution $u \in$ $W_{0}^{1,1}(\Omega)$ of

$$
\begin{cases}-\Delta u=\mu & \text { in } \Omega \\ u=0 & \text { on } \partial \Omega\end{cases}
$$

is a solution in the sense of Stampacchia of the same equation.

Proof. Let $\varphi \in W_{0}^{1,2}(\Omega) \cap C(\bar{\Omega})$ be such that $g:=\Delta \varphi \in C(\bar{\Omega})$. By the regularity result in [24, Theorem 1] (in the case $\Omega$ convex, see also [9, Theorem 1.4]) or by [25, Theorem 1] (if $\partial \Omega$ is of class $C^{1, \alpha}$ ), we have that $\varphi \in W_{0}^{1, \infty}(\Omega)$. Take a sequence $\left\{u_{k}\right\}$ in $C_{c}^{\infty}(\Omega)$ approximating $u$ in the $W^{1,1}$-norm. Since $\Omega$ satisfies an interior cone condition, it is possible to use [8, Theorem 2.1] to integrate by parts in order to obtain

$$
-\int_{\Omega} u_{k} \Delta \varphi=\int_{\Omega} \nabla u_{k} \nabla \varphi .
$$


Sending $k \rightarrow \infty$, we obtain

$$
-\int_{\Omega} u \Delta \varphi=\int_{\Omega} \nabla u \nabla \varphi
$$

Now take a sequence $\left\{\varphi_{k}\right\}$ in $C_{c}^{\infty}(\Omega)$ approximating $\varphi$ in the $W^{1, \infty}$-norm. Then we have

$$
\begin{aligned}
-\int_{\Omega} u \Delta \varphi & =\int_{\Omega} \nabla u \nabla \varphi=\lim _{k \rightarrow \infty} \int_{\Omega} \nabla u \nabla \varphi_{k} \\
& =\lim _{k \rightarrow \infty} \int_{\Omega} \varphi_{k} d \mu=\int_{\Omega} \varphi d \mu .
\end{aligned}
$$

Hence we obtain the claim.

Remark 4.4. If $\partial \Omega$ is of class $C^{1,1}$, it is not necessary to use [8, Theorem 2.1] in the proof of the previous proposition. In that case, standard regularity theory implies that actually $\varphi \in W^{2, p}(\Omega) \cap W_{0}^{1, p}(\Omega)$ (see [17, Theorem 9.15]), and so the classical integration by parts applies.

Corollary 4.5. Let $\Omega$ be a bounded, convex domain or a bounded domain whose boundary is of class $C^{1, \alpha}$ with $\alpha \in(0,1]$. If $u \in W_{0}^{1,1}(\Omega)$ is harmonic in $\Omega$ in distributional sense, then $u \equiv 0$.

Proof. By Proposition $4.3 u$ is a solution in the sense of Stampacchia of

$$
\begin{cases}-\Delta u=0 & \text { in } \Omega \\ u=0 & \text { on } \partial \Omega .\end{cases}
$$

By uniqueness Theorem 4.2 it follows $u \equiv 0$.

\section{The eigenvalue problem for the 1-biharmonic operator}

The aim of this section is to discuss Theorem 1.1; its proof will be divided into different steps.

In the following we will assume that $\Omega$ is convex, or that its boundary is of class $C^{1, \alpha}(0<\alpha \leq 1)$. Corollary 4.5 and Theorem 4.2 imply that, for each Radon measure $\mu$ with finite total variation, there exists exactly one distributional solution $u \in W_{0}^{1,1}(\Omega)$ of the problem

$$
\begin{cases}-\Delta u=\mu & \text { in } \Omega \\ u=0 & \text { on } \partial \Omega .\end{cases}
$$


Moreover, $u \in W_{0}^{1, r}(\Omega)$ for every $r \in\left[1, \frac{n}{n-1}\right)$ (see Theorem 4.2). Let us define

$$
\Lambda_{1,1}(\Omega):=\inf _{u \in B L_{0}(\Omega) \backslash\{0\}} \frac{|\Delta u|_{T}}{\|u\|_{1}} .
$$

In the next proposition we will show that the above infimum is attained for a function $u \in B L_{0}(\Omega)$.

Proposition 5.1. There exists a function $u \in B L_{0}(\Omega)$ such that

$$
\frac{|\Delta u|_{T}}{\|u\|_{1}}=\Lambda_{1,1}(\Omega) .
$$

Proof. Let $\left\{u_{k}\right\}$ be a minimizing sequence in $B L_{0}(\Omega)$ such that $\left\|u_{k}\right\|_{1}=1$. Since there exists a $M>0$ such that $\left|\Delta u_{k}\right|_{T} \leq M$, by Theorem 4.2 the sequence is uniformly bounded in $W_{0}^{1, r}(\Omega)$ for a fixed $r \in\left(1, \frac{n}{n-1}\right)$. So there exists a function $u \in W_{0}^{1, r}(\Omega)$ (and hence in $W_{0}^{1,1}(\Omega)$ ) such that, up to a subsequence, $u_{k} \rightarrow u$ weakly in $W_{0}^{1, r}(\Omega)$ and $u_{k} \rightarrow u$ strongly in $L^{1}(\Omega)$; this implies in particular that $\left\|u_{k}\right\|_{1}=1$. By Remark 2.1 we obtain the claim.

A minimizer $u$ can be seen as the first eigenfunction of the 1-biharmonic operator, where the associated Euler-Lagrange equation has to be interpreted in an appropriate sense. In fact, $u$ formally satisfies

$$
\begin{cases}\Delta\left(\frac{\Delta u}{|\Delta u|}\right)=\lambda \frac{u}{|u|} & \text { in } \Omega \\ u=0 & \text { on } \partial \Omega \\ \frac{\Delta u}{|\Delta u|}=0 & \text { on } \partial \Omega\end{cases}
$$

for $\lambda=\Lambda_{1,1}(\Omega)$. The last equation is formally obtained as a natural boundary condition which is similar to the usual Navier boundary condition $\Delta u=0$. However, the expressions $\frac{\Delta u}{|\Delta u|}$ and $\frac{u}{|u|}$ are undetermined for $\Delta u=0$ and $u=0$ respectively. To overcome this difficulty, we follow [20, Proposition 4.23] adapting the results to our situation. We define the extension of $|\Delta u|_{T}$ in the space $L^{n^{\prime}}(\Omega)$ for $n^{\prime}=\frac{n}{n-1}$ (observe that $B L_{0}(\Omega) \subseteq W_{0}^{1,1}(\Omega) \subseteq L^{\frac{n}{n-1}}(\Omega)$ )

$$
E(u):=\left\{\begin{aligned}
|\Delta u|_{T} \text { if } u \in B L_{0}(\Omega) \\
+\infty \text { if } u \in L^{n^{\prime}}(\Omega) \backslash B L_{0}(\Omega) .
\end{aligned}\right.
$$

The idea is to consider minimizers of $E$ constrained on the set $\left\{u \in L^{n^{\prime}}(\Omega) \mid\|u\|_{1}=1\right\}$. 
Proposition 5.2. Let $u \in B L_{0}(\Omega)$, and denote by $\partial E(u)$ the subdifferential of $E$ at $u$. Then $u^{*} \in \partial E(u)$ if and only if there exists $z \in W_{0}^{1,1}(\Omega) \cap L^{\infty}(\Omega)$ such that:

- $\|z\|_{\infty} \leq 1$

- $u^{*}=\Delta z \in L^{n}(\Omega)$ in distributional sense;

- $E(u)=\int_{\Omega} u \Delta z$.

Moreover, if $u \neq 0$, then $\|z\|_{\infty}=1$.

Proof. For the proof we can follow [20, Proposition 4.23] almost verbatim. Let us define

$$
M^{*}:=\left\{u^{*} \in L^{n}(\Omega) \mid u^{*}=\Delta z \text { for some } z \in W_{0}^{1,1}(\Omega) \cap L^{\infty}(\Omega),\|z\|_{\infty} \leq 1\right\} .
$$

Since in particular $u^{*} \in L^{1}(\Omega)$, by regularity we have that $M^{*}=M_{r}^{*}$ for every $r \in\left[1, n^{\prime}\right)$, where

$$
M_{r}^{*}:=\left\{u^{*} \in L^{n}(\Omega) \mid u^{*}=\Delta z \text { for some } z \in W_{0}^{1, r}(\Omega) \cap L^{\infty}(\Omega),\|z\|_{\infty} \leq 1\right\} .
$$

We want to show that $M^{*}$ is closed. To this end, fix $r \in\left(1, n^{\prime}\right)$ and take a sequence $\left\{u_{k}^{*}\right\}$ in $M^{*}$ such that $u_{k}^{*} \rightarrow u^{*}$ in $L^{n}(\Omega)$; since $M^{*}=M_{r}^{*}$, it is possible to find a sequence $\left\{z_{k}\right\}$ in $W_{0}^{1, r}(\Omega) \cap L^{\infty}(\Omega)$ with the property that $\left\|z_{k}\right\|_{\infty} \leq 1$ for every $k$, and that $u_{k}^{*}=\Delta z_{k}$ in distributional sense, which means

$$
\int_{\Omega} u_{k}^{*} \varphi=\int_{\Omega} z_{k} \Delta \varphi \text { for every } \varphi \in C_{c}^{\infty}(\Omega) .
$$

The sequence $\left\{z_{k}\right\}$ is then a distributional solution of the equation $\Delta v=u_{k}^{*}$ with Dirichlet boundary condition, bounded in $L^{\infty}(\Omega)$; by Proposition 4.3 each distributional solution $v$ to this problem is also a Stampacchia solution, so that, by Theorem 4.2, it satisfies $\|v\|_{W_{0}^{1, r}} \leq c(r)\left\|u_{k}^{*}\right\|_{1}$ for any $r \in\left[1, n^{\prime}\right.$ ) (or, alternatively, by [6, Theorem 8]); as a consequence, the sequence $\left\{z_{k}\right\}$ is bounded also in $W_{0}^{1, r}(\Omega)$, since $\left\{u_{k}^{*}\right\}$ is uniformly bounded in $L^{1}(\Omega)$. So there exists a function $z \in W_{0}^{1, r}(\Omega) \cap L^{\infty}(\Omega)$ such that, after passing to a subsequence,

$$
z_{k} \rightarrow z \text { in } W_{0}^{1, r}(\Omega), \quad z_{k} \rightarrow^{*} z \text { in } L^{\infty}(\Omega),
$$

which implies $\|z\|_{\infty} \leq \liminf _{k \rightarrow \infty}\left\|z_{k}\right\|_{\infty} \leq 1$ and

$$
\int_{\Omega} u^{*} \varphi=\int_{\Omega} z \Delta \varphi \text { for every } \varphi \in C_{c}^{\infty}(\Omega),
$$

which means that $u^{*}=\Delta z$ in distributional sense. Hence, $u^{*} \in M^{*}$.

Let $I_{M^{*}}: L^{n}(\Omega) \rightarrow \mathbb{R}$ be the function defined as

$$
I_{M^{*}}\left(u^{*}\right)= \begin{cases}0 & \text { if } u^{*} \in M^{*} \\ +\infty & \text { otherwise }\end{cases}
$$


The conjugate function to $I_{M^{*}}$ is given by

$$
I_{M^{*}}^{*}(u)=\sup _{u^{*} \in L^{p^{\prime}}(\Omega)}\left\{\int_{\Omega} u^{*} u-I_{M^{*}}\left(u^{*}\right)\right\}=\sup _{u^{*} \in M^{*}} \int_{\Omega} u^{*} u .
$$

Now take $u \in B L_{0}(\Omega)$ and $u^{*} \in M^{*}$; then there exists a sequence $\left\{u_{k}\right\}$ in $C^{\infty}(\Omega) \cap$ $B L_{0}(\Omega) \cap C(\bar{\Omega})$ such that $u_{k} \rightarrow u$ in $W_{0}^{1,1}(\Omega)$ (without loss of generality also in $\left.L^{n^{\prime}}(\Omega)\right)$ and $\left|\Delta u_{k}\right|_{T} \rightarrow|\Delta u|$ as $k \rightarrow \infty$. We have

$$
\begin{aligned}
\int_{\Omega} u^{*} u & =\int_{\Omega} \Delta z u=\lim _{k \rightarrow \infty} \int_{\Omega} \Delta z u_{k}=\lim _{k \rightarrow \infty} \int_{\Omega} z \Delta u_{k} \\
& \leq\|z\|_{\infty} \lim _{k \rightarrow \infty} \int_{\Omega}\left|\Delta u_{k}\right| \leq \lim _{k \rightarrow \infty} \int_{\Omega}\left|\Delta u_{k}\right|=\lim _{k \rightarrow \infty}\left|\Delta u_{k}\right|_{T}=|\Delta u|_{T} .
\end{aligned}
$$

Hence,

$$
I_{M^{*}}^{*}(u)=\sup _{u^{*} \in M^{*}} \int_{\Omega} u^{*} u \leq E(u) .
$$

Now we have

$$
\begin{aligned}
E(u) & =\sup \left\{\int_{\Omega} \nabla u \nabla \varphi \mid \quad \varphi \in C_{c}^{\infty}(\Omega),\|\varphi\|_{\infty} \leq 1\right\} \\
& =\sup \left\{\int_{\Omega} u \Delta \varphi \mid \quad \varphi \in C_{c}^{\infty}(\Omega),\|\varphi\|_{\infty} \leq 1\right\} \\
& \leq \sup \left\{\int_{\Omega} u \Delta z \mid \quad z \in W_{0}^{1,1}(\Omega) \cap L^{\infty}(\Omega), \Delta z \in L^{n}(\Omega),\|z\|_{\infty} \leq 1\right\} \\
& =\sup \left\{\int_{\Omega} u^{*} u \mid \quad u^{*} \in M^{*}\right\}=I_{M^{*}}^{*}(u) .
\end{aligned}
$$

Since the above inequality is true also for $u \in L^{n^{\prime}}(\Omega) \backslash B L_{0}(\Omega)$, we obtain

$$
I_{M^{*}}^{*}(u)=E(u)
$$

for every $u \in L^{n^{\prime}}(\Omega)$. $M^{*}$ is closed and convex and thus $I_{M^{*}}^{*}$ is convex and lower semicontinuous, which implies (see [15, Chapter 1, Propositions 3.1 and 5.1])

$$
I_{M^{*}}=\left(I_{M^{*}}^{*}\right)^{*}=E^{*} .
$$

By [15, Chapter 1, Proposition 5.1] one obtains that $u^{*} \in \partial E(u)$ if and only if

$$
\int_{\Omega} u u^{*}=E(u)+E^{*}\left(u^{*}\right)=E(u)+I_{M^{*}}\left(u^{*}\right),
$$

which implies that $u^{*} \in \partial E(u)$ if and only if $u^{*} \in M^{*}$ and $E(u)=\int_{\Omega} u^{*} u$, which is the claim. Moreover, if $u \neq 0$, then $E(u) \neq 0$ by Corollary 4.5 and hence $\|z\|_{\infty}=1$ from equation (5.1). 
Let us define $G: L^{n^{\prime}}(\Omega) \rightarrow \mathbb{R}, n^{\prime}=\frac{n}{n-1}$, as

$$
G(u):=\int_{\Omega}|u|
$$

For $u \in L^{n^{\prime}}(\Omega)$ one has that $u^{*} \in \partial G(u)$ if and only if

$$
u^{*} \in \operatorname{Sgn}(u)
$$

(see [20, Proposition 4.23]). We recall that $v \in \operatorname{Sgn}(u)$ if and only if:

- $v(x)=1$ if $u(x)>0$;

- $v(x)=-1$ if $u(x)<0$;

- $v(x) \in[-1,1]$ if $u(x)=0$.

We are now ready to characterize the first eigenfunctions of the 1-biharmonic operator (Theorem 1.1, part (ii)).

Proposition 5.3. Let $u \in B L_{0}(\Omega)$ be a minimizer of $E$ constrained to the set $\{u \in$ $\left.L^{n^{\prime}}(\Omega) \mid G(u)=1\right\}$. Then, for every measurable selection $s \in \operatorname{Sgn}(u)$ there exists $z \in W_{0}^{1,1}(\Omega) \cap L^{\infty}(\Omega)$ such that:

(1) $\|z\|_{\infty}=1$;

(2) $\Delta z \in L^{n}(\Omega)$ in distributional sense;

(3) $E(u)=|\Delta u|_{T}=\int_{\Omega} u \Delta z$;

(4) $\Delta z=\lambda$ s almost everywhere in $\Omega$, with $\lambda=E(u)$.

Proof. From [20, Proposition 6.4] (setting $\tilde{u}=-u$ ), it follows that for every $g^{*} \in$ $\partial G(u)$, there exists a $e^{*} \in \partial E(u)$ and a $\lambda \in \mathbb{R}$ such that $g^{*}=\lambda e^{*}$, which plays the role of a Lagrange multiplier rule in this non smooth setting. Multiplying both sides of the equality by $u$ and integrating on $\Omega$, one obtains that $\lambda=E(u)$. The claim easily follows if one remembers how the subdifferentials of $E$ and $G$ are characterized.

Remark 5.4. The function $s$ (respectively, $z$ ) in Proposition 5.3 can be seen as an interpretation of the possibly undetermined expression $\frac{u}{|u|}$ (respectively, $\frac{\Delta u}{|\Delta u|}$ ). Observe that the condition $z \in W_{0}^{1,1}(\Omega)$ allows to give a sense to the formal natural boundary condition $\frac{\Delta u}{|\Delta u|}=0$ on $\partial \Omega$.

Remark 5.5. In general, it is not true that $|\Delta| u||_{T} \leq|\Delta u|_{T}$. A counterexample is given by the function $u(x)=1-x$ defined on the interval $I:=[-1,1]$. Given a function $\varphi \in C_{c}^{\infty}(I)$ with $\|\varphi\|_{\infty} \leq 1$ one has

$$
\int_{-1}^{1} u^{\prime} \varphi^{\prime}=-\int_{-1}^{1} \varphi^{\prime}=\varphi(-1)-\varphi(1)=0
$$


so that $|\Delta u|_{T}=0$, while

$$
\int_{-1}^{1}|u|^{\prime} \varphi^{\prime}=-\int_{-1}^{0} \varphi^{\prime}+\int_{0}^{1} \varphi^{\prime}=\varphi(-1)-2 \varphi(0)+\varphi(1)=-2 \varphi(0)
$$

so that $|\Delta| u \|_{T}=2$.

The previous remark implies that it is impossible to prove the non-negativity of a first eigenfunction $u$ simply considering the Rayleigh quotient for the function $v:=|u|$, as can be done for the first eigenfunction of the Laplacian or even of the $p$ Laplacian. On the other hand, this approach does not work for eigenfunctions of the $p$-biharmonic operator $(p>1)$, since $u \in W^{2, p}(\Omega)$ does not imply $|u| \in W^{2, p}(\Omega)$. Nevertheless, the result holds true, as shown in the following result:

Proposition 5.6. Let $u \in B L_{0}(\Omega)$ be such that

$$
\frac{|\Delta u|_{T}}{\|u\|_{1}}=\min _{v \in B L_{0}(\Omega) \backslash\{0\}} \frac{|\Delta v|_{T}}{\|v\|_{1}} .
$$

Then $\mu:=-\Delta u$ is a positive Radon measure and $u$ is non-negative.

Proof. Suppose by contradiction that the Radon measure $\mu:=-\Delta u$ is not a positive measure. Let us consider the Jordan decomposition $\mu=\mu^{+}-\mu^{-}$, where $\mu^{+}$and $\mu^{-}$are nontrivial positive measures. Let $w$ and $z$ be the solutions of the problems

$$
\left\{\begin{array} { l l } 
{ - \Delta w = \mu ^ { + } } & { \text { in } \Omega } \\
{ w = 0 } & { \text { on } \partial \Omega , }
\end{array} \quad \left\{\begin{array}{ll}
-\Delta z=\mu^{-} & \text {in } \Omega \\
z=0 & \text { on } \partial \Omega
\end{array}\right.\right.
$$

Then $u=w-z$, and since $w, z \geq 0$ and $w, z \not \equiv 0$ by the maximum principle (see [38, Theorem 2.9]), it holds $\|u\|_{1}<\|w\|_{1}+\|z\|_{1}$. From [31, Theorem 6.13] one has

$$
|\Delta u|_{T}=|\mu|(\Omega)=\mu^{+}(\Omega)+\mu^{-}(\Omega)=\left|\mu^{+}\right|(\Omega)+\left|\mu^{-}\right|(\Omega)=|\Delta w|_{T}+|\Delta z|_{T} .
$$

But then

$$
\min \left\{\frac{|\Delta w|_{T}}{\|w\|_{1}}, \frac{|\Delta z|_{T}}{\|z\|_{1}}\right\} \leq \frac{|\Delta w|_{T}+|\Delta z|_{T}}{\|w\|_{1}+\|z\|_{1}}<\frac{|\Delta u|_{T}}{\|u\|_{1}},
$$

a contradiction to the definition of $u$. Hence, $u$ must be superharmonic (and hence non-negative).

Remark 5.7. We observe that a similar result was obtained in [14, Theorem 1.1] for the first eigenfunction of the $p$-biharmonic operator under Navier boundary conditions. 


\section{A physical interpretation for $\Lambda_{1,1}(\Omega)$}

This last section is devoted to the evaluation of the first eigenvalue of the 1-biharmonic operator for general domains. In order to state our next result, let us introduce the elastic torsion problem. Let $\Omega \subseteq \mathbb{R}^{n}$ be a bounded convex domain, or a bounded domain with boundary of class $C^{1, \alpha}(0<\alpha \leq 1)$, and consider the problem

$$
\begin{cases}-\Delta \psi=1 & \text { in } \Omega \\ \psi=0 & \text { on } \partial \Omega .\end{cases}
$$

If $n=2$ and the domain is simply connected, the problem is related to the well known elastic torsion problem, where $\psi$ is the torsion function of a beam with cross section $\Omega$; other physical applications for problem (6.1) can be found, for instance, in [21].

By standard arguments, $\psi$ is the unique classical solution of (6.1), is nonnegative and its maximum value $\psi_{M}(\Omega)=\|\psi\|_{\infty}$ is assumed in the interior of $\Omega$, thanks to the maximum principle; upper and lower bounds for $\psi_{M}(\Omega)$ are well known in the literature (see for instance [34, Chapter 6], [21, 29]). In the following we will prove that the maximum value $\psi_{M}(\Omega)$ of the torsion function is the reciprocal of the first eigenvalue of the 1-biharmonic operator, $\Lambda_{1,1}$ (see Theorem $1.2)$.

Proof of Theorem 1.2. Let $u \in B L_{0}(\Omega)$ be a minimizer for

$$
\Lambda_{1,1}(\Omega)=\min _{v \in B L_{0}(\Omega) \backslash\{0\}} \frac{|\Delta v|_{T}}{\|v\|_{1}} ;
$$

by Proposition 5.6, $u$ is a non-negative function, with $\mu=-\Delta u$ a positive Radon measure. By the divergence theorem, and recalling that $u=\psi=0$ on $\partial \Omega$

$$
\begin{aligned}
\|u\|_{1} & =\int_{\Omega} u d x=\int_{\Omega} u(-\Delta \psi) d x \\
& =\int_{\Omega} \nabla u \cdot \nabla \psi d x=\int_{\Omega} \psi d \mu \\
& \leq \psi_{M}(\Omega) \int_{\Omega} d \mu=\psi_{M}(\Omega)|\mu|(\Omega)=\psi_{M}(\Omega)|\Delta u|_{T}
\end{aligned}
$$

so that

$$
\frac{|\Delta u|_{T}}{\|u\|_{1}} \geq \frac{1}{\psi_{M}(\Omega)}
$$

We have then proved that

$$
\Lambda_{1,1}(\Omega)=\inf _{u \in B L_{0}(\Omega) \backslash\{0\}} \frac{|\Delta u|_{T}}{\|u\|_{1}} \geq \frac{1}{\psi_{M}(\Omega)} .
$$


Let us now prove the reverse inequality. Since $\partial \Omega$ is at least of class Lipschitz, there exists a unique Green function $G: \Omega \times \Omega \rightarrow \mathbb{R}, G \geq 0$ satisfying the problem

$$
\begin{cases}-\Delta G(z, x)=\delta_{z} & x \in \Omega \\ G(z, x)=0 & x \in \partial \Omega\end{cases}
$$

$\delta_{z}$ denoting the Dirac measure giving unit mass to the point $z$ (see [22, Theorem 1.2.8]). Let $z_{M}$ be a maximum point for $\psi$, i.e., $\psi\left(z_{M}\right)=\psi_{M}$, and set

$$
v(x)=G\left(z_{M}, x\right) .
$$

Then $v \in B L_{0}(\Omega)$, and $|\Delta v|_{T}=1$ by definition of the Green function; on the other hand, the function $\psi$ can be represented as

$$
\psi(z)=\int_{\Omega}(-\Delta \psi(x)) G(z, x) d x
$$

so that

$$
\begin{aligned}
\psi_{M}(\Omega) & =\psi\left(z_{M}\right)=\int_{\Omega}(-\Delta \psi(x)) G\left(z_{M}, x\right) d x \\
& =\int_{\Omega} v(x) d x=\int_{\Omega}|v(x)| d x \\
& =\|v\|_{1}
\end{aligned}
$$

thanks to the definition of $\psi$ and the positivity of the Green function. We have then proved that

$$
\frac{|\Delta v|_{T}}{\|v\|_{1}}=\frac{1}{\psi_{M}(\Omega)} .
$$

Statement (1.5) follows combining (6.3) with (6.4); furthermore, (6.4) proves that the function $v(x)=G\left(z_{M}, x\right)$ is an eigenfunction for $\Lambda_{1,1}$.

If $\Omega=B_{R}$ is a ball with radius $R$, then $\psi(x)=\frac{1}{2 n}\left(R^{2}-|x|^{2}\right)$ and therefore $\Lambda_{1,1}\left(B_{R}\right)=\frac{2 n}{R^{2}}$. Moreover, the solution of the problem

$$
\begin{cases}-\Delta u=\delta_{0} & \text { in } B_{R} \\ u=0 & \text { on } B_{R},\end{cases}
$$

is a first eigenfunction. Hence, in the 1-dimensional case, $u$ has the form

$$
u(r)=\frac{1}{2}(R-|r|) \quad \text { if } r \in(-R, R)
$$

whereas in the other cases

$$
u(r)= \begin{cases}\frac{1}{2 \pi} \log \left(\frac{R}{r}\right) & \text { if } n=2, \\ \frac{1}{n(n-2) \omega_{n}}\left(r^{2-n}-R^{2-n}\right) & \text { if } n \geq 3 .\end{cases}
$$


Remark 6.1. We verify that the function $u$ defined in (1.7) satisfies the conditions stated in Proposition 5.3. Indeed, since $u>0$ in $B_{R}$ we have that $s \equiv 1$ in $B_{R}$, so that the function $z$ must satisfy

$$
\begin{cases}\Delta z=\frac{2 n}{R^{2}} & \text { in } B_{R} \\ z=0 & \text { on } \partial B_{R} .\end{cases}
$$

It is easy to check that the function $z(x)=\frac{|x|^{2}}{R^{2}}-1$ is a solution of the above equation, which moreover satisfies $\|z\|_{\infty}=1$.

Remark 6.2. We observe that the first eigenfunction $u$ of the 1-biharmonic operator in a ball which we have found has a singularity at the origin for $n \geq 2$, and so it does not belong to $L^{\infty}(\Omega)$. Moreover, we have that $u \in W_{0}^{1, q}(\Omega)$ for $q \in\left[1, \frac{n}{n-1}\right)$, but $u \notin W_{0}^{1, \frac{n}{n-1}}(\Omega)$. This means that in general an eigenfunction does not enjoy more regularity than its "natural" regularity given by Theorem 4.2.

As a consequence of the previous proof, we have the following 1- dimensional inequality.

Corollary 6.3. Let $u \in W^{2,1}(a, b) \cap W_{0}^{1,1}(a, b)$. Then

$$
\int_{a}^{b}|u| \leq \frac{(b-a)^{2}}{8} \int_{a}^{b}\left|u^{\prime \prime}\right| .
$$

Proof. This is a direct consequence of Theorem 1.2 when $n=1$. Note that the sharp constant is not attained on $W^{2,1}(a, b) \cap W_{0}^{1,1}(a, b)$.

Remark 6.4. Corollary 6.3 improves the result that one could obtain by combining two optimal inequalities: on one hand, we have

$$
\int_{a}^{b}|u| \leq \frac{(b-a)}{2} \int_{a}^{b}\left|u^{\prime}\right|
$$

(see for instance [36]), while from the fact that

$$
\int_{a}^{b} u^{\prime}=u(b)-u(a)=0
$$

it follows from [1, Theorem 3.2] that

$$
\int_{a}^{b}\left|u^{\prime}\right| \leq \frac{(b-a)}{2} \int_{a}^{b}\left|u^{\prime \prime}\right| .
$$

Both inequalities are optimal, but their combination would give

$$
\int_{a}^{b}|u| \leq \frac{(b-a)^{2}}{4} \int_{a}^{b}\left|u^{\prime \prime}\right|
$$


where the constant is not optimal. This fact is essentially due to the non coincidence of the extremal functions of the two cited inequalities. Indeed, the extremal function for inequality (6.5)

$$
\int_{a}^{b}|u| \leq \frac{(b-a)}{2} \int_{a}^{b}\left|u^{\prime}\right|, \quad u \in W_{0}^{1,1}(a, b)
$$

is given by

$$
u_{1}(x)=\chi_{(a, b)}(x)
$$

which belongs to $B V(\mathbb{R})$, while the extremal function for the inequality

$$
\int_{a}^{b}|u-\bar{u}| \leq \frac{(b-a)}{2} \int_{a}^{b}\left|u^{\prime}\right|, \quad u \in W^{1,1}(a, b)
$$

(where $\bar{u}=\frac{1}{b-a} \int_{a}^{b} u$ ), which implies directly (6.6), is given by

$$
u_{2}(x)=\frac{1}{2} \chi_{\left(a, \frac{a+b}{2}\right)}(x)-\frac{1}{2} \chi_{\left(\frac{a+b}{2}, b\right)}(x) .
$$

Remark 6.5. Since the first eigenfunction of the Laplace operator with Dirichlet boundary condition belongs to $B L_{0}(\Omega)$ and satisfies the equation

$$
\begin{cases}-\Delta \varphi=\lambda_{1}(\Omega) \varphi & \text { in } \Omega \\ \varphi=0 & \text { on } \partial \Omega\end{cases}
$$

we obtain the following upper bound for $\Lambda_{1,1}(\Omega)$ :

$$
\Lambda_{1,1}(\Omega) \leq \lambda_{1}(\Omega)
$$

Let us remark that first eigenfunctions may not be unique, since we can find an eigenfunction for each maximum point of the torsion function for the domain $\Omega$. However, if the torsion function has only one maximum point (as is the case of convex domains), then the first eigenfunction is unique, as shown by the following result:

Proposition 6.6. Suppose that the solution $\psi$ of (6.1) admits only one maximum point $z_{M} \in \Omega$. Then, the solution of

$$
\begin{cases}-\Delta v=\delta_{z_{M}} & \text { in } \Omega \\ v=0 & \text { on } \partial \Omega\end{cases}
$$

is the only first eigenfunction of the 1-biharmonic operator. 
Proof. Let $u \in B L_{0}(\Omega)$ be a first eigenfunction satisfying $|\Delta u|_{T}=1$. Looking at the proof of Theorem 1.2, one notices that the equality sign must hold in (6.2). This implies in particular that

$$
\int_{\Omega} \psi d \mu=\psi_{M}(\Omega)
$$

where $\mu=-\Delta u$, and therefore $\mu$ must be supported in the set $\mathcal{M}:=\{x \in$ $\left.\Omega \mid \psi(x)=\psi_{M}\right\}$. If $\mathcal{M}=\left\{z_{M}\right\}$, this implies that $\mu=\delta_{z_{M}}$, and therefore the claim is proved.

Finally, we show that also for the 1-biharmonic operator the Faber-Krahn inequality holds true: among all domains with prescribed volume, the first eigenvalue $\Lambda_{1,1}$ is minimal for the ball.

Proof of Theorem 1.3. By Theorem 1.2, $\Lambda_{1,1}(\Omega)=\frac{1}{\psi_{M}(\Omega)}$ where $\psi$ is the torsion function, solution of (6.1), and $\psi_{M}(\Omega)=\max _{x \in \Omega} \psi(x)$. Let us denote with $\tilde{\psi}$ the torsion function associated to the symmetrized domain $\Omega^{\#}$, that is

$$
\begin{cases}-\Delta \widetilde{\psi}=1 & \text { in } \Omega^{\#} \\ \widetilde{\psi}=0 & \text { on } \partial \Omega^{\#} .\end{cases}
$$

Then, by Talenti's comparison principle [37, Theorem 1] $\psi^{\#} \leq \widetilde{\psi}$ where $\psi^{\#}$ denotes the radially symmetric decreasing rearrangement of $\psi$; hence

$$
\psi_{M}(\Omega)=\|\psi\|_{L^{\infty}(\Omega)}=\left\|\psi^{\#}\right\|_{L^{\infty}\left(\Omega^{\#}\right)} \leq\|\tilde{\psi}\|_{L^{\infty}\left(\Omega^{\#}\right)}=\psi_{M}\left(\Omega^{\#}\right)
$$

which implies directly the first part of the claim, while the second part follows from [23, Theorem 5.1].

Remark 6.7. By Theorem 1.2 and Theorem 1.3 we have

$$
\Lambda_{1,1}(\Omega) \geq \Lambda_{1,1}\left(\Omega^{\#}\right)=\frac{2 n}{R^{2}}
$$

where $R$ is the radius of the ball $\Omega^{\#}$ having the same measure as $\Omega$. Note that, under dilation, $\Lambda_{1,1}$ scales as follows

$$
\begin{aligned}
\Lambda_{1,1}(t \Omega) & =\min _{u \in B L_{0}(t \Omega)} \frac{|\Delta u|_{T}}{\|u\|_{1}}=\min _{v \in B L_{0}(\Omega)} \frac{t^{n-2}|\Delta v|_{T}}{t^{n}\|v\|_{1}} \\
& =\frac{1}{t^{2}} \min _{v \in B L_{0}(\Omega)} \frac{|\Delta v|_{T}}{\|v\|_{1}}=\frac{1}{t^{2}} \Lambda_{1,1}(\Omega)
\end{aligned}
$$

so that we obtain

$$
|\Omega|^{2 / n} \Lambda_{1,1}(\Omega) \geq\left|\Omega^{\#}\right|^{2 / n} \Lambda_{1,1}\left(\Omega^{\#}\right)=2 n \omega_{n}^{2 / n} .
$$


As a consequence, we recover the following upper bound for the maximum value of the torsion function

$$
\psi_{M}(\Omega)=\max _{x \in \Omega} \psi(x) \leq \frac{1}{2 n}\left(\frac{|\Omega|}{\omega_{n}}\right)^{2 / n}
$$

(see also $[28,30])$.

\section{References}

[1] G. Acosta and R. G. DuRÁn, An optimal Poincaré inequality in $L^{1}$ for convex domains, Proc. Amer. Math. Soc. 132 (2004), 195-202.

[2] R. A. Adams and J. J. F. Fournier, "Sobolev Spaces" (2 $2^{\text {nd }}$ ed.), Pure Appl. Math., Vol. 140, Academic Press, NY, 2003.

[3] L. Ambrosio, N. Fusco and D. Pallara, "Functions of Bounded Variation and Free Discontinuity Problems", Oxford University Press, 2000.

[4] L. BOCCARDO and T. GALLOUËT, Nonlinear elliptic equations with right-hand side measures, Commun. Partial Differential Equations 17 (1992), 641-655.

[5] L. BOCCARDO and T. GALLOUËT, Non-linear elliptic and parabolic equations involving measure data, J. Funct. Anal. 87 (1989), 149-169.

[6] H. BREZIS and W. STRAUSS, Semi-linear second-order elliptic equations in $L^{1}$, J. Math. Soc. Japan 25 (1973), 565-590.

[7] D. CASSANI, B. RUF and C. TARSI, Best costants in a borderline case of second order Moser type inequalities, Ann. Inst. Henri Poincaré, Anal. Non Linéaire 27 (2010), 73-93.

[8] G. Q. CHEN and H. FRID, On the theory of divergence-measure fields and its applications, Bull. Braz. Math. Soc. (N.S.) 32 (2001), 401-433.

[9] A. CIANCHI and V. G. MAZ'YA, Global Lipschitz regularity for a class of quasilinear elliptic equations, Commun. Partial Differential Equations 36 (2011), 100-133.

[10] S. Conti, D. FARACO and F. MAGGI, A new approach to counterexamples to $L^{1}$ estimates: Korn's inequality, geometric rigidity, and regularity for gradients of separately convex functions, Arch. Ration. Mech. Anal. 175 (2005), 287-300.

[11] G. Dal Maso, F. Murat, L. Orsina and A. Prignet, Renormalized solutions of elliptic equations with general measure data, Ann. Scuola Norm. Sup. Pisa Cl. Sci. (4) 28 (1999), 741-808.

[12] F. Demengel, Fonctions à hessien borné, Ann. Inst. Fourier (Grenoble) 34 (1984), 155190.

[13] F. Demengel, Some existence's results for noncoercive "1-Laplacian" operator, Asymptotic Anal. 43 (2005), 287-322.

[14] P. DRÁBEK and M. ÔTANI, Global bifurcation result for the p-biharmonic operator, Electron. J. Differential Equations 48 (2001), 1-19.

[15] I. Ekeland and R. TEMAM, "Convex Analysis and Variational Problems", North-Holland, Amsterdam, 1976.

[16] L. C. Evans, The 1-Laplacian, the $\infty$-Laplacian and differential games, Contemp. Math. 446 (2007), 245-254.

[17] D. Gilbarg and N. S. Trudinger, "Elliptic Partial Differential Equations of Second Order", Springer, 2001.

[18] E. Giusti, "Minimal Surfaces and Functions of Bounded Variation", Birkäuser, Boston, 1984.

[19] B. KAWOHL and V. FRIDMAN, Isoperimetric estimates for the first eigenvalue of the pLaplace operator and the Cheeger constant, Commentat. Math. Univ. Carol. 44 (2003), 659-667. 
[20] B. KAWOHL and F. SCHURICHT, Dirichlet problems for the 1-Laplace operator, including the eigenvalue problem, Commun. Contemp. Math. 9 (2007), 515-543.

[21] G. KEADY and A. MCNABB, Functions with constant Laplacian satisfying homogeneous Robin boundary conditions, IMA J. Appl. Math. 50 (1993), 205-224.

[22] C. E. KenIG, "Harmonic Analysis Techniques for Second Order Elliptic Boundary Value Problems", CBMS No83, American Mathematical Society, 1998.

[23] S. KeSAVAn, Some remarks on a result of Talenti, Ann. Scuola Norm. Sup. Pisa Cl. Sci. (4) 15 (1988), 453-465.

[24] O. LADYZHENSKAYA and N. URAL'TSEVA, A Lipschitz estimate at the boundary points for solutions of quasilinear equations of divergence form, Sibi. Mat. J. 28 (1987), 145-153.

[25] G. M. LIEBERMAN, Boundary regularity for solutions of degenerate elliptic equations, Nonlinear Anal. 12 (1988), 1203-1219.

[26] G. Mingione, The Calderón-Zygmund theory for elliptic problems with measure data, Ann. Sc. Norm. Super. Pisa Cl. Sci. (5) 6 (2007), 195-261.

[27] E. PARINI, The second eigenvalue of the p-Laplacian as p goes to 1, Int. J. Differential Equations. Article ID 984671, 23 pp. (2010).

[28] L. E. PAYNE, Isoperimetric inequalities and their applications, SIAM Rev. 9 (1967), 453488.

[29] L. E. PAYNE and G. A. PhILIPPIn, Isoperimetric inequalities in the torsion and clamped membrane problems for convex plane domains, SIAM J. Math. Anal. 14 (1983), 1154-1162.

[30] G. PólYA and G. SZEGÖ, "Isoperimetric Inequalities of Mathematical Physics", Princeton University Press, Princeton, NJ, 1951.

[31] W. Rudin, "Real and Complex Analysis" (3 ${ }^{\text {rd }}$ ed.), McGraw-Hill, New York, 1987.

[32] F. SCHURICHT, An alternative derivation of the eigenvalue equation for the 1-Laplace operator, Arch. Math. 87 (2006), 572-577.

[33] J. SERRIn, Pathological solutions of elliptic differential equations, Ann. Scuola Norm. Sup. Pisa Cl. Sci. (3) 18 (1964), 385-387.

[34] R. SPERB, "Maximum Principles and their Applications", Academic Press, New York, 1981.

[35] G. Stampacchia, Le problème de Dirichlet pour des équations elliptiques du second ordre à coéfficients discontinus, Ann. Inst. Fourier (Grenoble) 15 (1965), 189-258.

[36] G. TAlenti, Best constant in Sobolev inequality, Ann. Mat. Pura Appl. 110 (1976), 353372.

[37] G. TAlenti, Elliptic equations and rearrangements, Ann. Scuola Norm. Sup. Pisa Cl. Sci. (4) 3 (1976), 697-718.

[38] L. VÉRON, Elliptic equations involving measures, In: "Handbook of Differential Equations: Stationary Partial Differential Equations", M. Chipot and P. Quittner (eds.) 1, 2004, 593712 .

Ceremade, Université Paris-Dauphine

Place du Maréchal de Lattre de Tassigny

75775 Paris CEDEX 16, France

parini@ceremade.dauphine.fr

Dipartimento di Matematica "Federigo Enriques"

Università degli Studi di Milano

via Saldini 50, 20133 Milano, Italia

bernhard.ruf@unimi.it

cristina.tarsi@unimi.it 